\title{
Are we getting closer to identifying the best follow-up and management after Fontan completion?
}

\author{
Yves d'Udekem, MD, PhD, ${ }^{\mathrm{a}, \mathrm{b}, \mathrm{c}}$ Jean-Benoit Thambo, MD, PhD, ${ }^{\mathrm{d}, \mathrm{e}}$ \\ Ajay J. Iyengar, MBBS, PhD, FRACS, ${ }^{a, b, c}$ and Jack Rychik, MD
}

Feature Editor's Note-There is a rapidly growing population of patients with Fontan circulation worldwide. These patients require thorough lifelong medical management. As these patients get older, they may require further complex surgical interventions, including ventricular assist device implantation and heart, or multiorgan, transplantation. The expert opinion published herein summarizes very timely current guidelines on the best management of these challenging patients.

\section{Igor E. Konstantinov, MD, PhD, FRACS}

The American Heart Association (AHA) has now published a Statement entitled "Evaluation and Management of the Child and Adult With Fontan Circulation." It is an extraordinary document concentrating a huge breadth of knowledge on this topic and providing some directions to enlighten best practices in this population.

The original idea to create such a document emerged at the time of a Fontan "think tank" meeting organized in Bordeaux, France, in June 2016. ${ }^{2}$ Seventeen of the world's thinkers and experts gathered in this historical location where the Fontan procedure was performed in 1968 , motivated by the realization that (1) this population far outlived their expected survival, (2) the maintenance of this abnormal physiology over decades had

From the a Department of Cardiac Surgery, Royal Children's Hospital, Melbourne, Australia; ' Department of Pediatrics, University of Melbourne, Australia; ${ }^{\mathrm{C}} \mathrm{Heart}$ Research Group, Murdoch Children's Research Institute, Melbourne, Australia; ${ }^{\mathrm{d}}$ Pediatric and Congenital Cardiology Unit, Bordeaux University Hospital,

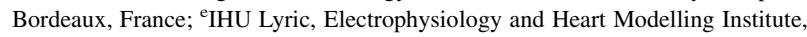
Fondation Bordeaux Université, F-33600 Pessac, France; and ${ }^{\mathrm{f}}$ Division of Pediatric Cardiology, and Cardiac Center at The Children's Hospital of Philadelphia, The Perelman School of Medicine at the University of Pennsylvania, Philadelphia, Pa.

This work was supported by a National Health and Medical Research Council (NHMRC) Partnership grant (1076849). The Murdoch Children's Research Institute received support through the Victorian Government's Operational Infrastructure Support Program. Dr d'Udekem is a NHMRC Clinician Practitioner Fellow (1082186).

Received for publication Nov 14, 2019; revisions received March 18, 2020; accepted for publication March 20, 2020; available ahead of print July 4, 2020.

Address for reprints: Yves d'Udekem, MD, PhD, Department of Cardiac Surgery, Royal Children's Hospital, Flemington Rd, Parkville, 3052 Australia (E-mail: yves.dudekem@rch.org.au).

J Thorac Cardiovasc Surg 2021;162:222-7

$0022-5223 / \$ 36.00$

Copyright (c) 2020 by The American Association for Thoracic Surgery

https://doi.org/10.1016/j.jtcvs.2020.03.186

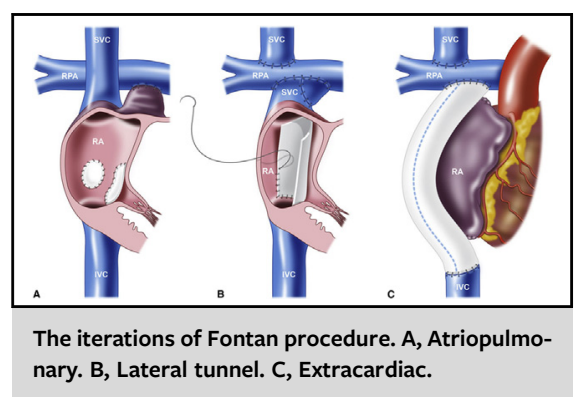

CENTRAL MESSAGE

Recently published in Circulation, the AHA Statement "Evaluation and Management of the Child and Adult With Fontan Circulation" is a landmark in the evolution of care of this special population.

This Invited Expert Opinion provides a perspec tive on the following paper: Circulation 2019;140(6):e234-e284. https://doi.org/10.1161/CIR 0000000000000696

multiple unexpected consequences, and (3) there is currently a lack of clarity on how to best care for and follow these patients.

Because of the lack of abundant high-quality evidence, it was impossible at this stage to prescribe formal guidelines for the management and treatment of this growing population Nevertheless, the information provides good rationale with consensus-based best practice suggestions to help practitioners identify ways to organize follow-up care. With a total of 51 pages and more than 400 references, this document is difficult to digest, and a snapshot of the new and most relevant findings is provided here.

The historical development of the procedure and the physiology of the Fontan circulation will be familiar to a surgical readership. We know today that those who undergo the extracardiac and the lateral-tunnel Fontan have a survival advantage compared with those with the original atriopulmonary Fontan (Figure 1). Current expectations of 
30-year survival greater than $80 \%$ for those operated today with the current version of the procedure are well recognized. ${ }^{3,4}$ This document clarifies better than ever before the current state of knowledge on the complications of the Fontan circulation.

\section{LATE IMPACT AND POTENTIAL CHALLENGES WITH A FONTAN CIRCULATION Circulatory Failure}

Failure of the Fontan circulation includes not only classical pump failure-diastolic or systolic ventricular dysfunction-but also a status of failure characterized by increased venous pressures and symptoms suggestive of right heart failure. These 2 modes of failure may exist concurrently or in isolation with some patients suffering from ascites and edema despite a relatively preserved ventricular function. Overall, the rate of failure is still estimated to be $1 \%$ per year of follow-up. It is now understood that ventricular function is initially preserved but declines progressively in the subsequent decades. ${ }^{5}$

\section{Cyanosis}

Cyanosis is often part of the life of those with a Fontan circulation because of the presence of a fenestration, ventilation-perfusion mismatch inherent to this nonpulsatile circulation and the development of abnormal vascular intrapulmonary connections, especially in patients with heterotaxia. Because of mild cyanosis, hemoglobin levels are often elevated, and to preserve this compensatory mechanism maintenance of good iron stores is important. ${ }^{6}$

\section{Atrioventricular (AV) Valve Regurgitation}

There is clear evidence that patients with singleventricle anatomy have a high risk of AV valve regurgitation developing over the course of their life, with common AV and tricuspid valves being more prone to failure. Structural valve abnormalities, annular dilatation, and reduced contractility can all contribute to this phenomenon in addition to the abnormally high pressure and volume load that these hearts may face in the early stages of life. More than moderate regurgitation is associated with a risk of failure of the Fontan circulation, and repairing these lesions should be performed before or, at the latest, during Fontan procedure. ${ }^{7}$ Despite their frequent use, there is no evidence that afterloadreducing therapies are of any benefit, and caution in their use is recommended because of their capacity to decrease cardiac output. ${ }^{8}$

\section{Arrhythmia}

The burden of this frequent complication has been long recognized. It is thought that the differences in the rates of arrhythmia reported are related to variations in follow-up length and that arrhythmia will ultimately affect more than one half of patients, with a risk of sudden death between $0.15 \%$ and $0.2 \%$ per year. ${ }^{9}$ Besides arrhythmias intrinsically related to particular conditions (such as double
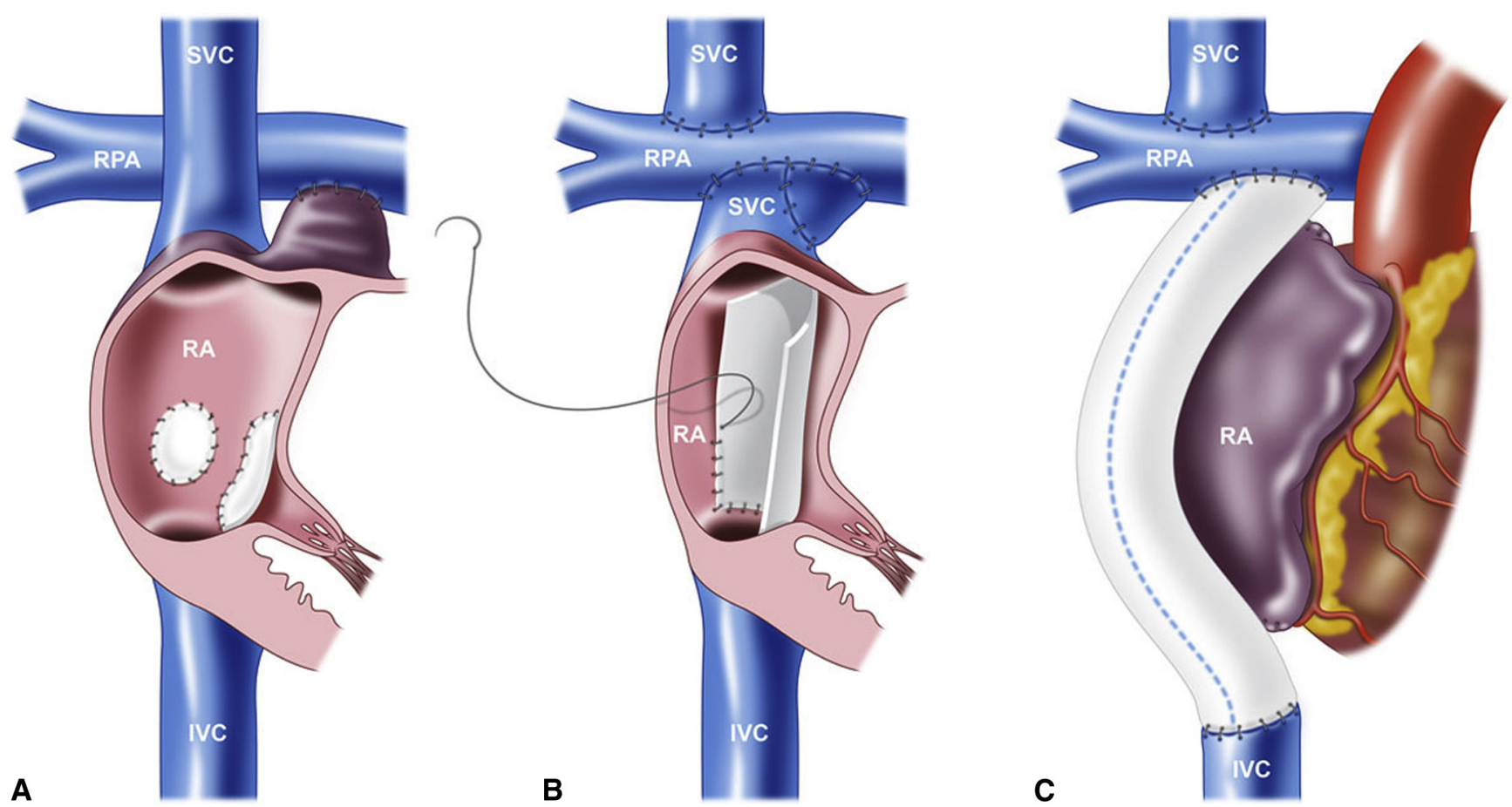

FIGURE 1. Various techniques of the Fontan procedure. A, Atriopulmonary connection. B, Lateral tunnel. C, Extracardiac conduit. SVC, Superior vena cava; $R P A$, right pulmonary artery; $R A$, right atrium; $I V C$, inferior vena cava. Reproduced with permission. ${ }^{1}$ 
discordance or Ebstein disease), identified risk factors are older age at Fontan, atriopulmonary type of repair, preoperative and early postoperative tachycardia, moderate or greater AV valve regurgitation, and longer follow-up. Sinus-node dysfunction is common, and chronotropic incompetence during exercise has been identified in the majority, impairing exercise capacity. The implantation of a pacemaker is foreseen in more than $10 \%$ of patients in their first 2 decades, contributing to a risk of death, transplantation, Fontan failure, decreased quality of life, and impaired ventricular function. ${ }^{10}$ The onset of arrhythmia should prompt hemodynamic assessment and intervention. Because of the high rate of recurrence with medical therapy, ablation should be considered early, even though its success rate is inferior to ablation in other congenital heart diseases. Patients with atriopulmonary Fontan and dilated atria should be considered for a combination of ablation and conversion surgery or directed toward evaluation for transplantation.

\section{Protein-Losing Enteropathy (PLE)}

PLE is thought to affect $5 \%$ to $12 \%$ of patients. ${ }^{11}$ Abnormal intestinal blood flow, deficits in surface proteins, and inflammation seem to influence the occurrence of this extremely severe complication, but it seems that the main contributing factor is the increased load on the abdominal lymphatic circulation. ${ }^{12}$ It is possible that individual anatomical variations in the lymphatic circulation also influence this risk. ${ }^{13}$ PLE may persist for years and be subclinical between flares, being responsible for multiple imbalances such as coagulation abnormalities, hypoalbuminemia resulting in hypocalcemia with bone loss, and lymphopenia. The diagnosis is provided by elevated stool $\alpha-1$ antitrypsin in a 24-hour collection or in a single specimen when associated with edema and hypoalbuminemia. The presence of PLE mandates a full hemodynamic assessment and intervention on all correctable lesions. Treatments include a combination of diuretics, especially spironolactone, albumin replacement or immunoglobulin administration, and dietary modifications. Enteral corticosteroids (particularly budesonide) have been shown to provide symptomatic relief. Exogenous unfractionated heparin has been reported to give remission in selected cases. Direct intervention on the lymphatic channels between the liver and the intestine seems promising and derivative procedures have now been attempted. ${ }^{14}$ The ultimate treatment of this complication remains orthotopic heart transplantation.

\section{Plastic Bronchitis}

Plastic bronchitis sits in the same spectrum of lymphatic diseases resulting from the Fontan circulation. Secondary to an increased load of this circulation, lymph leaks in the bronchial lumen and following an inflammatory reaction, fibrin cross-linking occurs within the lymph, which coagulates in a thick cast. Expectorations of these rubbery casts is typical of the condition and, at the extreme, can lead to asphyxiation and death. After elimination of potential hemodynamic causes increasing pressure on the venous and the lymphatic circulation, the treatment is mainly symptomatic. Mucolytics and nebulized administration of tissue plasminogen activator have been shown to provide symptomatic relief. Catheter-based interventions to block lymphatic communications with the airways have been effective in expert hands. Transplantation is again the ultimate cure of this complication.

\section{Liver Fibrosis and Cirrhosis}

It has now become obvious that all patients with a Fontan circulation present with some degree of liver fibrosis that increases with time. ${ }^{15}$ This complication has been termed "Fontan-associated liver disease" (FALD). Mild elevations of liver enzymes are commonly seen. The most-feared liver complication is the occurrence of hepatocellular carcinoma, whose prevalence seems to be around $1 \%$ at this stage but has been seen in patients as young as 12 years. ${ }^{16}$ Cirrhosis is extremely rare. There is a lack of correlation between the extent of fibrosis observed and the functional impact on the liver of these patients. The usual functional assessment tools of the liver function may not be adapted to FALD.

\section{Renal dysfunction}

Renal dysfunction develops as a result of repeated insults from surgeries, medications, or interventions. Given their long-term exposure to low cardiac output and greater venous pressures, it is no surprise that they show signs of impairment. In early adulthood, one half show some signs of mild renal dysfunction, and about one third have proteinuria. ${ }^{17}$ These findings are concerning, but at this stage there are no signs renal dysfunction affects long-term survival.

\section{Bone Health and Somatic Growth}

By adolescence, most patients have a deficit in lean muscle mass and vitamin D deficiency with features of secondary hyperparathyroidism. ${ }^{18}$ After the Fontan operation, patients have been identified to have delay in linear growth and abnormal bone structure, which could potentially expose them to osteopenia and an increased risk of bone fracture.

\section{Obesity}

The Fontan population has a lower incidence of obesity than the general population. This incidence seems to increase from childhood to adulthood, with rates varying from $10 \%$ to up to $50 \% .{ }^{19}$ The impact of obesity has not been fully investigated but might have an extremely deleterious impact on the Fontan circulation. 


\section{Brain and Neurocognitive Function}

There is a constellation of reasons for these patients to have abnormal brain development spanning from innate factors to multiple surgeries and risk of repeated microthrombi. It is no surprise that the vast majority show abnormalities on magnetic resonance imaging. On average, they have a lower IQ, lower numerical aptitude and reading comprehension, and impaired visuospatial skills. ${ }^{20}$ Executive dysfunction and attention-deficit disorders are concerning because they can affect well-being later in life. The bleak picture provided by the existing cross-sectional studies do not, however, enlighten the fact that many of these patients are highly functioning individuals.

\section{Psychosocial Challenges and Advanced Care Planning}

Two-thirds of adolescents with a Fontan circulation receive a psychiatric diagnosis such as anxiety, attentiondeficit/hyperactivity disorder, or mood disorder. ${ }^{21}$ As all studies point to greater difficulties in their young adult life, centers are encouraged to establish programs to support and improve these patients' cognitive and psychological outcomes.

Despite the knowledge that most of these patients will face premature death, less than $15 \%$ of those surveyed had ever participated in an advanced care planning discussion. ${ }^{22}$ These conversations are especially important, as deterioration of the Fontan circulation occurs rapidly, unexpectedly, and often during a hospital admission, where all interventions can be potentially attempted but not always desired by patients and their families.

\section{HOW TO MONITOR AND MANAGE PATIENTS WITH A FONTAN CIRCULATION Monitoring}

There is a large variation in follow-up pathways. It has become exquisitely difficult to ascertain best follow-up strategies because it is now clear that surveillance should not be limited to the cardiac condition but should also be extended to the late extracardiac consequences of this circulation. Among the vast array of possible investigations, the clinician faces difficulties making choices because the yield of these diverse investigations is still unknown. Once complications are actually identified, the best ways to treat them or prevent their progression are still unclear. Because of this lack of evidence, it was not possible to provide clear guidelines, so the group of experts responsible for this statement built a "surveillance toolkit" to provide guidance. The cardiac investigations proposed apply in turn to childhood, adolescence and adulthood. The extracardiac investigations are classified as basic, in-depth, or investigational to instruct the reader. One rapidly realizes that a regular comprehensive assessment will not be accessible to all, outside of super-specialized clinics because of the lack of time, workforce, or money. However, this extraordinary summary provides a perspective on the breadth of investigations of interest and focuses the attention on the different systems that at the minimum require observation and intermittent check-ups.

\section{Care of Adults With a Fontan Circulation}

As this population ages, a larger proportion made up of adults who are probably one of the most challenging groups to follow and support. Their mortality and morbidities increase with time and it is now realized that, compared with those with normal hearts, these patients face a much greater morbidity when faced with other medical events. Contraception and pregnancy provide particular challenges. Pregnancy has been successfully achieved in a number of women with a Fontan circulation but at the cost of a high rate of potential fetal loss and maternal complications. ${ }^{23}$

\section{Exercise Training}

Exercise training is increasingly becoming recognized to bring benefits, with leg muscle resistance training and inspiratory muscle training seeming superior to aerobic training. ${ }^{24,25}$ The recorded improvements in cardiac output and in quality of life seem to justify their recommendations because of the rarity of adverse events reported after exercise.

\section{Pharmacologic Management}

We lack strong evidence to identify the best pharmacologic therapies, and practitioners tend to use their own conviction to determine medical therapy. As an example, angiotensin-converting enzyme inhibitors are used in many without any evidence of benefit or harm. There is good evidence that patients are prone to thrombotic events, and thromboprophylaxis is recommended but there is still equipoise in the argument of choosing between aspirin and warfarin. ${ }^{26}$ From the current consensus statement, ${ }^{1}$ it is seems reasonable to recommend aspirin in all patients and reserve warfarin for those with additional risk factors, such as previous thrombotic episodes, PLE, or older age. Several trials are underway to elucidate whether longterm treatment with pulmonary vasodilators is beneficial.

\section{Heart Transplantation and Mechanical Circulatory Support}

Indications, timing and management of heart transplantation remain unclear. The conflict is often between offering transplantation early in patients who may continue for many years with a reasonable quality of life, or late in patients who have by then accumulated so many comorbidities that their outcomes after transplantation are compromised. In the last decade, outcomes after transplantation for pediatric patients with a Fontan circulation has improved to 
the point of being similar to those for dilated cardiomyopathy. No reliable adult figures have been reported. Everything points to the need to have these surgeries performed in highly specialized centers. Mechanical circulatory support is becoming feasible with conventional devices and a line of research is under way to provide dedicated support to the right-sided circulation.

The AHA Statement identifies the gaps in knowledge that need to be bridged to advance the care of this population. More specifically, there is a need for better understanding of the physiology of the Fontan circulation, the ability to apply individual risk scoring, and the description of late neurodevelopmental outcomes.

One cannot read this document without being daunted by the long list of complications. We were concerned that patients and their families reading this Statement would find it unnecessarily depressing. We have therefore tried to contextualize everything in a series of "take-away messages."

The Fontan population is growing rapidly and soon the majority of those with a Fontan circulation will be adults. The existence of this AHA Statement is a testament to the fact that survival after the Fontan is far better than it was ever expected to be. We all know many patients who lead active lives and are well integrated into society. However, the grown-up patients we are currently encountering are the results of our first attempts and we are likely to see better outcomes in future generations of survivors. We are just initiating the first interventional trials in this population. There is no doubt that our surgical community will be instrumental to further improving the outcomes of these patients by performing and fine-tuning interventions to optimize their circulation, treat their complications such as PLE, and provide them with mechanical circulatory support.

Above all, this Statement is a remarkable testimony to the newly acquired relevance of this population. We have produced an in-depth document on patients undergoing the Fontan procedure published in one of the most important journals of our specialty. The essential meaning of these 51 pages is the recognition by our specialty that these patients are here to stay, so a communal effort is now required to help them achieve a normal quality and duration of life.

\section{Conflict of Interest Statement}

Dr d'Udekem is a consultant for Merck Sharpe \& Dohme and Actelion. Dr Iyengar has received an Educational Grant from Edwards Lifesciences. All other authors reported no conflicts of interest.

The Journal policy requires editors and reviewers to disclose conflicts of interest and to decline handling or reviewing manuscripts for which they may have a conflict of interest. The editors and reviewers of this article have no conflicts of interest.

\section{References}

1. Rychik J, Atz AM, Celermajer DS, Deal BJ, Gatzoulis MA, Gewillig MH, et al. Evaluation and management of the child and adult with Fontan circulation: a scientific statement from the American Heart Association. Circulation. 2019;140: e234-84.

2. d'Udekem Y, Rychik J. Towards the goal of achieving a normal duration and quality of life after Fontan operation: creation of the international Fontan interest group (I-FIG), an international collaborative initiative dedicated to improving outcomes. Int J Cardiol. 2017;245:131-4.

3. d'Udekem Y, Iyengar AJ, Galati JC, Forsdick V, Weintraub RG, Wheaton GR, et al. Redefining expectations of long-term survival after the Fontan procedure: twenty-five years of follow-up from the entire population of Australia and New Zealand. Circulation. 2014;130(11 suppl 1):S32-8.

4. Schilling C, Dalziel K, Nunn R, Du Plessis K, Shi WY, Celermajer D, et al. The Fontan epidemic: population projections from the Australia and New Zealand Fontan registry. Int J Cardiol. 2016;219:14-9.

5. Atz AM, Zak V, Mahony L, Uzark K, D'agincourt N, Goldberg DJ, et al. Longitudinal outcomes of patients with single ventricle after the Fontan procedure. $J$ Am Coll Cardiol. 2017;69:2735-44.

6. Tomkiewicz-Pajak L, Plazak W, Kolcz J, Pajak J, Kopec G, Dluzniewska N, et al. Iron deficiency and hematological changes in adult patients after Fontan operation. J Cardiol. 2014;64:384-9.

7. King G, Ayer J, Celermajer D, Pajak J, Kopec G, Dluzniewska N, et al. Atrioventricular valve failure in Fontan palliation. J Am Coll Cardiol. 2019;73: 810-22.

8. Hsu DT, Zak V, Mahony L, Sleeper LA, Atz AM, Levine JC, et al. Enalapril in infants with single ventricle: results of a multicenter randomized trial. Circulation. 2010;122:333-40.

9. Pundi KN, Pundi KN, Johnson JN, Dearani JA, Li Z, Driscoll DJ, et al. Sudden cardiac death and late arrhythmias after the Fontan operation. Congenit Heart Dis. 2017;12:17-23.

10. Williams RV, Travison T, Kaltman JR, Cecchin F, Colan SD, Idriss SF, et al. Comparison of Fontan survivors with and without pacemakers: a report from the pediatric heart network Fontan cross-sectional study. Congenit Heart Dis. 2013;8:32-9.

11. John AS, Johnson JA, Khan M, Driscoll DJ, Warnes CA, Cetta F. Clinical outcomes and improved survival in patients with protein-losing enteropathy after the Fontan operation. J Am Coll Cardiol. 2014;64:54-62.

12. Dori Y, Keller MS, Rome JJ, Gillespie MJ, Glatz AC, Dodds K, et al. Percutaneous lymphatic embolization of abnormal pulmonary lymphatic flow as treatment of plastic bronchitis in patients with congenital heart disease. Circulation. 2016;133:1160-70.

13. Itkin M, Piccoli DA, Nadolski G, Rychik J, DeWitt A, Pinto E, et al. Proteinlosing enteropathy in patients with congenital heart disease. J Am Coll Cardiol. 2017;69:2929-37.

14. Itkin M, Piccoli DA, Nadolski G, Rychik J, DeWitt A, Pinto E, et al. Proteinlosing enteropathy in patients with congenital heart disease. J Am Coll Cardiol. 2017;69:2929-37.

15. Goldberg DJ, Surrey LF, Glatz AC, Dodds K, O’Byrne ML, Lin HC, et al. Hepatic fibrosis is universal following Fontan operation, and severity is associated with time from surgery: a liver biopsy and hemodynamic study. J Am Heart Assoc. 2017;6:e004809.

16. Egbe AC, Poterucha JT, Warnes CA, Connolly HM, Baskar S, Ginde S, et al. He patocellular carcinoma after Fontan operation: multicenter case series. Circula tion. 2018;138:746-8.

17. Wilson TG, d'Udekem Y, Winlaw DS, Cordina RL, Celermajer DS, Wheaton FR, et al. Hepatic and renal end-organ damage in the Fontan circulation: a report from the Australian and New Zealand Fontan registry. Int J Cardiol. 2018;273:100-7.

18. Avitabile CM, Leonard MB, Zemel BS, Brodsky JL, Lee D, Dodds K, et al. Lean mass deficits, vitamin D status and exercise capacity in children and young adults after Fontan palliation. Heart. 2014;100:1702-7.

19. Chung ST, Hong B, Patterson L, Petit CJ, Ham JN. High overweight and obesity in Fontan patients: a 20-year history. Pediatr Cardiol. 2016;37:192-200. 
20. Bellinger DC, Watson CG, Rivkin MJ, Robertson RL, Roberts AE, Stopp C, et al. Neuropsychological status and structural brain imaging in adolescents with single ventricle who underwent the Fontan procedure. J Am Heart Assoc. 2015;4: e002302.

21. DeMaso DR, Calderon J, Taylor GA, Holland JE, Stopp C, White MT, et al. Psychiatric disorders in adolescents with single ventricle congenital heart disease. Pediatrics. 2017;139:e20162241.

22. Tobler D, Greutmann M, Colman JM, Greutmann-Yantiri M, Librach SL, Kovacs AH. Knowledge of and preference for advance care planning by adults with congenital heart disease. Am J Cardiol. 2012;109:1797-800.

23. Garcia Ropero A, Baskar S, Roos Hesselink JW, Girnius A, Zentner D, Swan L, et al. Pregnancy in women with a Fontan circulation: a systematic review of the literature. Circ Cardiovasc Qual Outcomes. 2018;11:e004575.

24. Duppen N, Etnel JR, Spaans L, Takken T, van den Berg-Emons RJ, Boersma E, et al. Does exercise training improve cardiopulmonary fitness and daily physical activity in children and young adults with corrected tetralogy of Fallot or Fontan circulation? A randomized controlled trial. Am Heart J. 2015;170:606-14.

25. Cordina RL, O'Meagher S, Karmali A, Rae CL, Liess C, Kemp GJ, et al. Resistance training improves cardiac output, exercise capacity and tolerance to positive airway pressure in Fontan physiology. Int J Cardiol. 2013;168: 780-8.

26. Marrone C, Galasso G, Piccolo R, de Leva F, Paladini R, Piscione F, et al. Antiplatelet versus anticoagulation therapy after extracardiac conduit Fontan: a systematic review and meta-analysis. Pediatr Cardiol. 2011;32:32-9.

Key Words: AHA scientific statements, Fontan procedure, congenital heart defects, clinical guidelines, univentricular circulation 